Soz.- Präventivmed. 42 (1997) 105-113

$0303-8408 / 97 / 0200105-09 \$ 1.50+0.20 / 0$

(C) Birkhäuser Verlag, Basel, 1997

Helmut Knolle

Institut für Sozial- und Präventivmedizin der Universität Bern

\title{
Zunehmender Heroin- und Kokainkonsum in der Schweiz seit 1990: Anwendung eines verallgemeinerten Poisson-Ansatzes auf Anzeigedaten
}

\begin{abstract}
Zusammenfassung
Die Prävalenz des Konsums harter illegaler Drogen wird mit den üblichen Surveymethoden weit unterschätzt Deshalb sind, wenn geeignete Daten vorliegen oder erhoben werden können, höhere statistische Methoden vorzuziehen, die auf der Zählung won einmaligen und widerholten Kontakten der Drogenkonsumierenden mit Institutionen beruhen. Hier wird ein erweiterter Poisson-Ansatz verwendet, um die Zah/ der Konsumenten von Heroin undloder Kokain in der Schweiz für jedes Jahr von 1990 bis 1993 zu schätzen, wobel zwischen Drogenkonsumenten mit geringer und höherer Anzeigerate unterschieden wird. Die Ergebnisse deuten auf einen Zuwachs der Konsumenten von Heroin und Kokain um ca. $50 \%$ im Zeitraum 1990-1993.
\end{abstract}

Der Konsum illegaler Drogen und seine verschiedenen Begleiterscheinungen haben in der Schweiz eine sehr kontroverse Debatte ausgelöst. Die eine Seite hält Kokain und Heroin, aber teilweise auch die sogenannten weichen Drogen für „Elixiere des Teufels", um den Titel eines Romans von E.T.A. Hoffmann zu zitieren, und fordert die Eindämmung des Drogenkonsums durch verstärkte Repression und abstinenzorientierte Therapieangebote für Abhängige. Die andere Seite warnt hingegen vor einer Dramatisierung des Problems und vor der gesellschaftlichen Ausgrenzung der drogenkonsumierenden Menschen und fordert eine Gleichstellung der bisher illegalen
Sozial- und Präventivmedizin 
Versuch, mit einem ziemlich willkürlich angenommenen Korrekturfaktor zu einer annähernd realistischen Zahl zu kommen, wäre nur dann verdienstvoll, wenn es keine besseren Möglichkeiten gäbe. Aber schon seit geraumer Zeit gibt es bessere Methoden, und diese werden auch zunehmend in der Drogenepidemiologie angewendet ${ }^{3-5}$.

Der zugrunde liegende Ansatz kann auf folgende Weise illustriert werden. Ein Statistiker, der fünf Tage lang an einem Kongress in X teilnimmt und an jedem Morgen ein Taxi benutzt, möchte zum Zeitvertreib schätzen, wie viele Taxifahrer es in X gibt. Bei 5 Fahrten hat er nur 4 verschiedene Chauffeure kennengelernt, denn am Freitag traf er zufällig auf den gleichen wie am Mittwoch. Er macht nun folgende Überlegung sowie einige kleine Berechnungen: Es gibt mindestens 4 Taxifahrer in X. Wenn es nicht mehr als 4 gäbe, dann hätte das Ereignis, dass ich bei 4 Fahrten jedesmal einen anderen treffe, die Wahrscheinlichkeit $0,75 \times 0,5 \times 25=0,09375$. Wenn es 5 gäbe, dann hätte das Ereignis, dass ich viermal einen anderen und beim fünften $\mathrm{Mal}$ einen schon bekannten Fahrer treffe, die Wahrscheinlichkeit $0,8 \times$ $0,6 \times 0,4 \times 0,8=0,1536$. So rechnet er weiter und findet, dass die Wahrscheinlichkeit des eingetretenen Ereignisses bei 8 Taxifahrern am grössten ist. Sein Ergebnis ist also die Zahl 8. Das Prinzip, aus der Wiederholungszahl von Kontakten auf die Grösse einer Population zu schliessen, liegt vielen neueren Arbeiten zur Drogenepidemiologie zugrunde.

Die Erkennung von wiederholten Kontakten setzt die Identifizierung der kontaktierten Personen voraus. Sie muss daher auf Datenschutzbestimmungen Rücksicht nehmen oder erfordert doch in jedem Fall zusätzlichen Arbeitsaufwand der Institutionen, die solche Kontakte registrieren. Vor die- sem Hintergrund wäre eine statistische Methode, die lediglich auf routinemässig publizierte amtliche Statistiken zurückgreift, von besonderem Wert. In der Schweiz werden die polizeilichen Anzeigen wegen Verstosses gegen das Betäubungsmittelgesetz (BetmG) seit 1974 vom Bundesamt für Polizeiwesen zentral registriert, und Auszählungen der anonymisierten Daten werden jährlich in der Schweizerischen Betäubungsmittelstatistik veröffentlicht. Diese enthält Tabellen mit Auszählungen nach Deliktart (Konsum, Handel, Schmuggel), Substanz, Alter, Geschlecht usw. Besonders nützlich für die Schätzung der Prävalenz und der Inzidenz des Konsums illegaler Drogen ist die Gliederung nach Rückfälligkeit, d.h. nach dem Kriterium, ob eine Person zum ersten oder wiederholten Mal wegen Verstoss gegen das BetmG angezeigt wird.

Bekanntlich hat die Zahl der registrierten BetmG-Anzeigen pro Jahr seit 1974 fast ununterbrochen zugenommen, besonders auffallend in den Jahren 1979-1983 und 1990-1993. Dies wurde vom Bundesamt für Statistik zunächst als Artefakt, der durch zunehmende Verfolgungsintensität bedingt sei, interpretiert ${ }^{6}$. Abklärungen zu dieser Frage in Zusammenarbeit mit Polizeistellen wurden anscheinend nie unternommen. In einer kürzlich erschienenen Publikation ${ }^{7}$ konnte jedoch, ausgehend von der amtlichen Betäubungsmittelstatistik, die Zahl der Konsumierenden von (allen) illegalen Drogen in der Schweiz für die Jahre 1977 bis 1990 geschätzt werden, wobei nicht nur die absolute Zahl aller Anzeigen, sondern auch der Anteil der Erstanzeigen in die Berechnungen einging. Mit einem Ansatz, der im Sinne von Wickens ${ }^{8}$ als system dynamics approach zu bezeichnen wäre, wurde gezeigt, dass der Zuwachs der Anzeigen wegen Konsums illegaler Drogen von 1977 bis 1990 in der Hauptsache auf einen massiven Anstieg der Zahl der Konsumenten und nur beiläufig auf einen geringen Anstieg der Verfolgungsintensität schliessen lässt.

Im folgenden soll nun der Konsum von Heroin und Kokain im Zeitraum 1990-1993 untersucht werden. Hierbei wird jedoch eine andere Methode als in der vorangehenden Publikation angewendet, da für die Jahre 1990 bis 1993 und teilweise auch für 1994 detailliertere Daten vorliegen. Das Bundesamt für Statistik publizierte nämlich zunächst für die Jahre 1990-1993 und ein Jahr später auch für 1994 die Zahl der Personen mit $1,2, \ldots, 6$ und mehr als 6 Anzeigen wegen Verstoss gegen das BetmG im gleichen Jahr ${ }^{9}$. Ausgehend von diesen Daten wurde bereits die Zahl der Personen, die dem Risiko einer polizeilichen Anzeige wegen Konsums illegaler Drogen ausgesetzt sind, für die Jahre 1990 bis 1994 geschätzt ${ }^{10}$. Wie in ${ }^{7}$, so sind auch hier die Konsumenten von Cannabisprodukten eingeschlossen. Es zeigte sich zwar eine Erhöhung des Risikos, aber ebenso ein Anstieg der Zahl der Konsumenten, nämlich von ca. 89000 in 1990 auf ca. 110000 in 1994. Eine ähnliche Datenbasis, jedoch auf den Konsum von Heroin und Kokain bezogen, ist in Tabelle 1 zusammengefasst. Auf diesen Daten beruhen die folgenden Analysen (Tabelle 1).

\section{Methoden}

Fang-Rückfang-Methode nach Peterson

Bereits im 18. Jahrhundert hat Laplace die Bevölkerung Frankreichs mit einer Methode geschätzt, die heute wegen ihrer häufigen Anwendung in der Populationsbiologie meist als Fang-Rückfang-Methode bezeichnet wird. Bei ihrer einfachsten Variante wird zweimal eine $\mathrm{Zu}$ - 


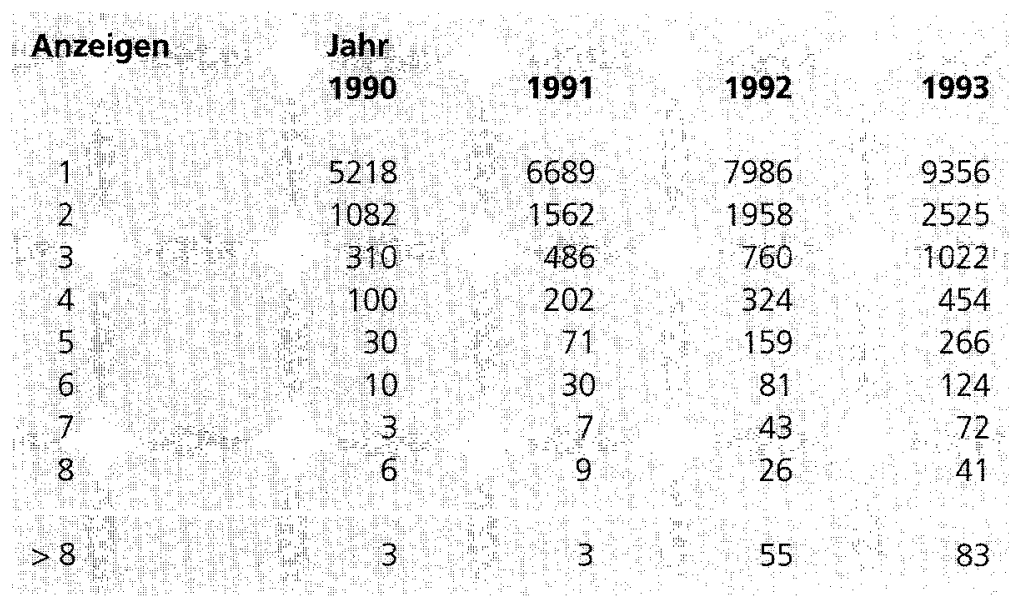

Tabelle 1. Zahl der Personen, die im entsprechenden Jahr 1, 2, ., 8 und mehr als $8 \mathrm{mal}$ wegen Konsums von Heroin undloder Kokain angezeigt worden sind (Quelle: BFS, auf Anfrage).

fallsstichprobe aus der Population gezogen. Alle Tiere der ersten Stichpobe werden markiert und dann wieder freigelassen. In der zweiten Stichprobe werden die markierten Tiere gesondert gezählt. So findet man drei Zahlen, nämlich

$\mathrm{a}=$ Grösse der 1. Stichprobe,

$\mathrm{b}=$ Grösse der 2. Stichprobe,

$c=$ Grösse der Überlappung beider Stichproben,

und erhält die Grösse der Population durch die Formel

$\mathrm{N}=\mathrm{ab} / \mathrm{c}$ (Peterson-Formel).

Die Fangwahrscheinlichkeit ist $\mathrm{a} / \mathrm{N}$ für die erste und $\mathrm{b} / \mathrm{N}$ für die zweite Stichprobe. Aus aktuellem Anlass (s.u.) muss hier betont werden, dass diese Fangwahrscheinlichkeiten durchaus verschieden sein können. Vorausgesetzt wird lediglich, dass beim zweiten Fang markierte und unmarkierte Tiere mit gleicher Wahrscheinlichkeit gefangen werden. Sei zum Beispiel $\mathrm{N}=1000$ und $\mathrm{a}=100, \mathrm{~b}=200$. Bis auf Zufallseffekte muss dann $\mathrm{c}=20$ sein, und die Formel würde im Mittel den richtigen Wert 1000 ergeben, da der Faktor 2 ebenso im Nenner wie im Zähler auftritt.
Bei Anwendungen in den Sozialwissenschaften werden die benötigten Daten geliefert von einer oder mehreren Institutionen, welche Personen aus der zu schätzenden Population namentlich oder anonym mit einem Codewort in einer Liste registrieren. An die Stelle der Markierung und Zählung von markierten Tieren tritt dann die Identifizierung von Personen, die auf mehreren Listen erscheinen.

Die Formel von Peterson setzt allerdings Bedingungen voraus, die in der gesellschaftlichen Wirklichkeit häufig nicht oder nur annähernd erfülllt sind. Insbesondere soll die Population homogen und geschlossen, und die zweite soll unabhängig von der ersten Registrierung sein. Nichterfüllung der Annahmen führt zu einer Verzerrung (bias) des Schätzergebnisses. Eine Sensitivitätsanalyse hat gezeigt, dass die Heterogenität der Population bezüglich der Registrierungswahrscheinlichkeit eine Unterschätzung, wohingegen die Fluktuation (Ein- und Austritte) eine Überschätzung der wahren Populationsgrösse nach sich zieht $^{11}$. Man kann also damit rechnen, dass diese beiden Effekte sich kompensieren oder zumindest ab- schwächen. Trotzdem sollte man den Effekt der Fluktuation dadurch klein halten, dass man das Zeitintervall, in dem die Stichproben gezogen werden, nicht mehr als nötig ausdehnt. Ein Intervall von 3 Monaten wie in einer spanischen Studie ${ }^{5}$ ist wahrscheinlich besser als das Intervall von 12 Monaten, das in der Schweiz gewählt wurde ${ }^{12}$.

\section{Der Poisson-Ansatz}

In den frühen 90er Jahren wurde die Zahl der Konsumenten von Heroin und/oder Kokain auf ca. 30000 geschätzt $^{2}$. Für das Jahr 1990, aber nicht für die folgenden Jahre, wird diese Schätzung im folgenden bestätigt werden. Auf 30000 Personen kamen also im Jahr 1990 laut Tabelle 1 rund 9000 Anzeigen. Das bedeutet, dass eine Anzeige wegen Konsums illegaler Drogen, auf das Individuum bezogen, ein relativ seltenes Ereignis ist. Selbst wenn man bei Personen, deren Hauptdroge Heroin oder Kokain ist, die Anzeigen wegen Konsums von Haschisch u. ä. hinzunimmt. Dies geht übrigens auch aus einer Publikation hervor, die ganz besonders die Zunahme der polizeilichen Repression in den letzten Jahren betont ${ }^{12}$.

Für die statistische Analyse von seltenen Ereignissen bietet sich die Poisson-Verteilung an ${ }^{13}$. Eine Poisson-Verteilung liegt immer dann vor, wenn viele Personen die gleiche Chance haben, ein wiederholbares Zufallsereignis $\mathrm{zu}$ erleben, und wenn ihre Chance durch bereits eingetretene Ereignisse nicht verändert wird. Das Gesetz von Poisson besagt, dass die Wahrscheinlichkeit $\mathrm{p}_{\mathrm{i}}$ für i-maliges Eintreten des Ereignisses mit den folgenden Formel berechnet werden kann:

$\mathrm{p}_{0}=\mathrm{e}^{-\alpha}$
$\mathrm{p}_{\mathrm{i}}=\left(\alpha^{\mathrm{i}} / \mathrm{i} !\right) \mathrm{e}^{-\alpha} \quad(\mathrm{i}=1,2, \ldots)$

Hierbei ist definitionsgemäss $\mathrm{i} !=$ $1 \times 2 \times \ldots \times$ i. Bei der gestutzten 
(engl. truncated) Poisson-Verteilung wird $\mathrm{p}_{\mathrm{i}}$ durch $1-\mathrm{p}_{0}$ dividiert. Dies ergibt die Wahrscheinlichkeit für i-maliges Eintreten unter der Bedingung, dass das Ereignis mindestens $1 \mathrm{mal}$ eintritt. Zur Schätzung der „Dunkelziffer“ $\mathrm{p}_{0}$ können Daten wie die in Tabelle 1 in vielen Fällen mit der gestutzten PoissonVerteilung analysiert werden ${ }^{14}$. Der Versuch, die Poisson-Verteilung an die Daten der Tabelle 1 anzupassen, führt jedoch zu enttäuschenden Ergebnissen. Die Schätzung des Poisson-Parameters $\alpha$ und der Populationsgrösse $\mathrm{N}$ nach der maximum-likelihood-Methode (vgl. Anhang) mit anschliessender Berechnung von $\mathrm{Np}_{\mathrm{i}}(\mathrm{i}=1,2, \ldots, 8)$ führt zu Werten, die von den $\mathrm{Da}$ ten sehr stark abweichen (Tabelle 2). Daraus kann zunächst nur geschlossen werden, dass nicht alle Voraussetzungen, die dem Poisson-Modell zugrunde liegen, erfüllt sind. Man muss daher ein Modell suchen, das die Daten besser erklären kann. Gegebenenfalls kann zusätzliches empirisches Material Hinweise zur Verbesserung des Modells geben. Deshalb soll jetzt zunächst der Entstehungsprozess der Anzeigenstatistik mit den wichtigsten Details modelliert werden.

Wir nehmen an, dass die Polizei die Drogenkonsumenten nur an öffentlich zugänglichen Orten und ohne Fixierung auf bestimmte Personen verfolgt, und dass eine Anzeige keinen oder höchstens einen dreimonatigen Freiheitsentzug (die Höchststrafe für reinen Konsum) zur Folge hat. Weiter nehmen wir an, dass es in der Population der Drogenkonsumenten keine Hierarchien gibt, die es einigen erlauben würden, sich auf Kosten anderer der Verhaftung zu entziehen. Schliesslich benötigen wir vorübergehend eine Voraussetzung, die, wenn sie erfüllt wäre, die oben beschriebene Anwendung der Poisson-Verteilung rechtfertigen würde. Dies ist die Voraussetzung, dass die Zeitspanne, während der sie sich dem Zugriff der Polizei aussetzen (Expositionszeit), für alle Drogenkonsumenten gleich ist.

Wenn nun in einem Zeitpunkt, in dem die offene Drogenszene einer Stadt von der Polizei umstellt wird, der Bruchteil p der Drogenkonsumenten präsent ist, von dem wiederum der Bruchteil $r$ angezeigt wird, und wenn dieser Vorgang sich n-mal in einem Jahr wiederholt, dann hat die Wahrscheinlichkeit, genau $k$-mal $(k=0,1, \ldots, n)$ angezeigt zu werden, eine Binomialverteilung mit den Parametern $\mathrm{n}$ und rp. Diese kann aber für grosses $n$ (bei kleinem rp) durch eine Poisson-Verteilung mit dem Parameter $\alpha=$ nrp ersetzt werden. Wir bezeichnen $\alpha$ als Anzeigerate. Sie ist das Produkt aus drei Faktoren, die vom Lebensstil der Drogenkonsumenten (Präsenz p), von der Strategie der Polizei (Zahl der Einsätze n), beziehungsweise von der Interaktion zwischen Polizei und Drogenkonsumenten (r) abhängen. Falls sich später zeigen sollte, dass die Anzeigerate gestiegen ist, so kann daraus allein noch nicht auf eine Intensivierung der Repression geschlossen werden.

Die obige Analyse gibt nun auch einen Hinweis zur Frage, was die Ursache der starken Abweichung der Daten von der Poisson-Verteilung sein könnte. Die individuelle
Präsenz- oder Expositionsdauer hat in der Population der Drogenkonsumenten sicher eine grosse Variationsbreite. Der Bereich kurzer Expositionsdauer wird noch erweitert durch Personen mit vorübergehenden Abstinenzperioden, die dazu führen, dass sie sich nicht während 12 , sondern nur während $3,4,5 \ldots$ Monaten regelmässig exponieren. Dies alles hat zur Folge, dass es nicht nur eine Anzeigerate $\alpha$ gibt, die für alle Drogenkonsumenten gleich ist, sondern eine Verteilung von Anzeigeraten. Der einfachste Weg, eine solche Verteilung in das Modell zu integrieren, führt zur negativen Binomialverteilung, die schon von Kendall auf eine Statistik von Arbeitsunfällen angewendet wurde ${ }^{15}$. Aber auch diese Verteilung, die bereits von zwei Parametern abhängt, lässt sich nicht hinreichend gut an die Daten der Tabelle 1 anpassen. Nach längeren Versuchen hat sich gezeigt, dass eine verallgemeinerte Poisson-Verteilung mit drei Parametern diese Anforderung erfüllen kann (vgl. Anhang). Dabei wird die Population der Heroin- und Kokainkonsumenten fiktiv in zwei Gruppen mit verschiedenen mittleren Anzeigeraten aufgeteilt, und auch innerhalb beider Gruppen ist die Rate variabel. Es muss noch diskutiert werden, ob dieser Trennung in zwei Gruppen eine reale

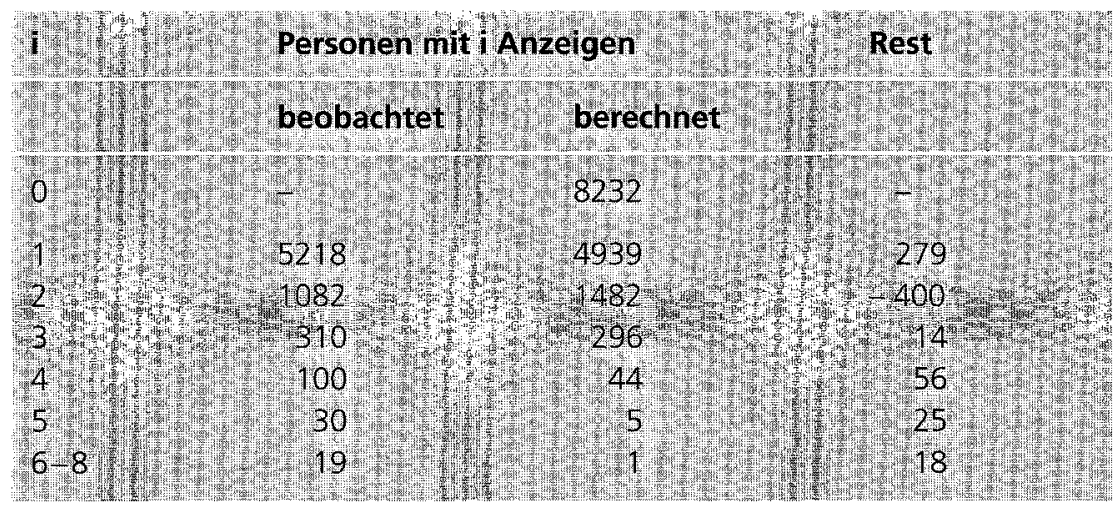

Tabelle 2. Mit der Poisson-Verteilung berechnete Zahl der Personen mit $0,1,2,3,4,5$ und 6-8 Anzeigen für $N=15000, \alpha=0,6$. Die Werte von $\alpha$ und $N$ wurden aus den Daten des Jahres 1990 geschätzt (vgl. Anhang). 


\begin{tabular}{cccccc}
\hline Jahr & $\mathbf{N}$ & $\mathbf{f}$ & $\boldsymbol{\alpha}$ & $\boldsymbol{\beta}$ & $\boldsymbol{\alpha}+(\mathbf{1}-\mathbf{f}) \boldsymbol{\beta}$ \\
\hline 1990 & & & & & \\
1991 & 29500 & 0,89 & 0,20 & 1,1 & 0,30 \\
1992 & 35000 & 0,88 & 0,23 & 1,4 & 0,36 \\
1993 & 40000 & 0,89 & 0,26 & 1,9 & 0,43 \\
& 44000 & 0,88 & 0,30 & 2,1 & 0,51
\end{tabular}

Tabelle 3. Geschätzte Zahl der Konsumenten ( $N$ ), Anteil der Gruppe mit niedriger Anzeigerate (f), mittlere Anzeigerate in jeder Gruppe ( $\alpha$ und $\beta$ ) und mittlere Anzeigerate in der ganzen Population.

Bedeutung zukommt. Sie erfüilt jedenfalls im Rahmen dieses Modells ihre Aufgabe, die Varianz der Anzeigeraten beträchtlich $\mathrm{zu}$ vergrössern.

\section{Ergebnisse}

Tabelle 3 zeigt für jedes Jahr von 1990 bis 1993 eine geschätzte Zahl der Heroin- und Kokainkonsumenten sowie die Parameterwerte, mit welchen die beste Anpassung an die Daten erreicht werden konnte. Dabei werden die folgenden Bezeichnungen verwendet:

$\mathrm{N}=$ Zahl der regelmässigen Konsumenten von Heroin und/ oder Kokain

$\mathrm{f}=$ Anteil der Subpopulation mit niedrigem Anzeigerisiko

$\alpha=$ niedrige Anzeigerate

$\beta=$ hohe Anzeigerate

Diese Ergebnisse bestätigen einerseits die Vermutung steigender Anzeigeraten. Aber auch die Zahl der Konsumenten ist in 3 Jahren beträchtlich angestiegen, nämlich um ca. $50 \%$. Der Unterschied in den Anzeigeraten der beiden Gruppen ist frappierend und fordert dazu auf, den entsprechenden Unterschied im Verhalten der Drogenkonsumenten näher zu untersuchen.

In Tabelle 4 werden die berechneten Werte mit den Daten verglichen. Bei der Beurteilung der Reste muss man im Auge behalten, dass die Anzahl der Parameter nicht beliebig erhöht werden darf. Vielmehr muss die Güte der Anpassung in einem ausgewogenen Verhältnis zur Anzahl der ge-

\begin{tabular}{|c|c|c|c|c|c|c|c|c|}
\hline & & 1 & 2 & 3 & 4 & 5 & 6 & $7-8$ \\
\hline \multirow[t]{4}{*}{1990} & beob. & 5218 & 1082 & 310 & 100 & 30 & 10 & 9 \\
\hline & berechn. & 5231 & 1096 & 312 & 107 & 37 & 12 & 5 \\
\hline & Rest & -13 & -14 & -2 & -7 & -7 & -2 & 4 \\
\hline & $x^{2}=5,36$ & & & & & & & \\
\hline \multirow[t]{4}{*}{1991} & beob. & 6689 & 1562 & 486 & 202 & 71 & 30 & 16 \\
\hline & berechn. & 6724 & 1588 & 515 & 202 & 81 & 31 & 15 \\
\hline & Rest & -35 & -26 & -29 & -0 & -10 & -1 & 1 \\
\hline & $x^{2}=3,45$ & & & & & & & \\
\hline \multirow[t]{4}{*}{1992} & beob & 7986 & 1958 & 760 & 324 & 159 & 81 & 69 \\
\hline & berechn. & 7903 & 1975 & 741 & 360 & 180 & 86 & 57 \\
\hline & Rest & 3 & -17 & 19 & -36 & -21 & -5 & 12 \\
\hline & $x^{2}=10,47$ & & & & & & & \\
\hline \multirow[t]{4}{*}{1993} & beob. & 9356 & 2525 & 1022 & 454 & 266 & 124 & 113 \\
\hline & berech. & 9405 & 2554 & 988 & 501 & 265 & 135 & 97 \\
\hline & Rest & -49 & -29 & 34 & -47 & 1 & -11 & 16 \\
\hline & $x^{2}=9,52$ & & & & & & & \\
\hline
\end{tabular}

Tabelle 4. Beobachtete und berechnete Zahl von Personen mit 1, 2, ., 6, und 7-8 Anzeigen im gleichen Jahr mit Resten und Pearson-Statistik $X^{2}$ gemäss Gleichung (A8). $X^{2}$ ist verteilt wie chi ${ }^{2}$ mit 3 Freiheitsgraden, also liegt der $95 \%$-Wert bei 7,8 und der $99 \%$-Wert bei 11,3 . 
schätzten Parameter stehen, d.h. die Pearson-Statistik $X^{2}$ muss kleiner sein als der $95 \%$ oder der 99\%-Punkt des chi $^{2}$ mit der entsprechenden Zahl von Freiheitsgraden. Im mathematischen Anhang wird gezeigt, dass dieses Ziel mit dem Zwei-Gruppen-Modell erreicht wird.

\section{Diskussion}

Frühere Analysen kamen in bemerkenswerter Übereinstimmung zu der Aussage, dass die Zahl der Konsumenten von Heroin/Kokain in der Schweiz seit 1990 stabil sei und rund 30000 betrage. Die Annahme, die zu dieser Konkordanz führten, sind jedoch durch die Fakten ungenügend begründet. Auch die Zitierung von Studien aus verschiedenen Jahren, die auf eine annähernd konstante Prävalenz des einmaligen Konsums (Lebenszeitprävalenz) hindeuten, ist als Beweis der These, dass der aktuelle Konsum stagniert, unzureichend.

Eine leicht zu handhabende Alternative zu der hier vorgeschlagenen Verallgemeinerung der PoissonMethode ist die Peterson-Methode. Wie am Anfang schon ausgeführt wurde, hat der Anstieg der Anzeigerate seit 1990, dem im Modell von Peterson eine Erhöhung der Fangwahrscheinlichkeit beim zweiten Fang entsprechen würde, keinen verzerrenden Effekt auf die Peterson-Schätzung. Tabelle 5 zeigt die von Estermann erhaltenen Schätzungen mit der Peterson-Methode ${ }^{16}$. Die dort angegebenen Konfidenzintervalle $(95 \%)$ halten sich im Bereich von $\pm 3 \%$ und zeigen keine Überlappungen, auch nicht für zusammenliegende Zeitintervalle. Die absoluten Zahlen liegen deutlich tiefer als in Tabelle 3, aber ein steigender Trend ist auch hier eindeutig erkennbar. Trotzdem wiederholt der Autor seine frühere These
„Mehr Repression - gleich viel Konsumenten" in einem zusammenfassenden Schlusswort, wo es heisst: „Auffällig ist, dass sich in dem kurzen Zeitraum 1990 bis 1994 die Zahl der Anzeigen fast verdreifacht und die Zahl der angezeigten Personen fast verdoppelt hat, die Zahl der Konsumierenden hingegen keine nachweisbare signifikante Veränderung erfuhr" ${ }^{\prime 17}$. Dies steht in Widerspruch zu den am gleichen Ort präsentierten Rechenergebnissen, die hier zum Vergleich zitiert worden sind.

In der gleichen Publikation wird auch der Versuch gemacht, die Zahl der Heroin- und Kokainkonsumenten für jedes Jahr von 1990 bis 1994 mit der Poisson-Verteilung zu schätzen. Wie zu erwarten findet der Autor, dass dieser Ansatz mit den Daten unvereinbar ist. Die Folgerung, die er daraus zieht, wird allerdings nicht weiter begründet:

„Die Anpassung einer Poisson-Verteilung ergibt allerdings hohe positive Residuen im Bereich von mehr als zwei Anzeigen pro Jahr: Ist eine Person mindestens zweimal angezeigt worden, steigt mit jeder Anzeige die Wahrscheinlichkeit einer weiteren" 18 . Der Autor zieht also die Heterogenität der Population an dieser Stelle nicht in Betracht und postuliert stattdessen, einem in der drogenpolitischen Diskussion oft gehörten Argumentationsmuster folgend, eine Kettenreaktion der Kriminalisierung, die sich nach jeder Anzeige weiter beschleunigt. Aber wie aus Tabelle 4 ersichtlich ist, führt der verallgemeinerte PoissonAnsatz, der die unterschiedlichen Anzeigeraten in Rechnung stellt, $\mathrm{zu}$ annehmbaren Residuen mit meist negativen Vorzeichen. Das Modell einer Kettenreaktion der Kriminalisierung und Verelendung folgt also nicht zwingend aus den Daten - ganz abgesezen davon, dass die zweite Anzeige im Jahr X keineswegs die zweite Anzeige im Leben eines Drogenkonsumenten sein muss.
In einer Analyse des Heroin- und Kokainkonsums in den frühen $90 \mathrm{er}$ Jahren in der Schweiz darf schliesslich auch der Hinweis auf die Methadonabgabe nicht fehlen. Die Zahl der Süchtigen, die unter ärztlicher Aufsicht Heroin durch Methadon substituieren, ist von 4800 in 1989 auf etwa 14000 in 1994 gestiegen $^{19}$. Insoweit die Methadonbezieher auf gleichzeitigen illegalen Drogenkonsum verzichten, fallen sie aus den hier vorgelegten Schätzungen heraus. Der Anstieg des Konsums von Opiaten (incl. Methadon) und Kokain ist also noch stärker als die Analyse der Verzeigungsdaten allein erkennen lässt.

Es stellt sich natürlich die Frage, ob der Anstieg der Prävalenz des Konsums durch eine Zunahme der Einstiege (Inzidenz), durch längere Dauer der Drogenkarrieren oder durch beides bedingt ist. Mit einer Methode, die unabhängig von jeder Kenntnis der Prävalenz den Trend der Inzidenz sichtbar macht, kann gezeigt werden, dass die Inzidenz des Konsums von Heroin und/oder Kokain im Zeitraum 1990/1991 erneut, wie schon 10 Jahre zuvor, angestiegen ist ${ }^{20}$.

In der vorliegenden Arbeit wurde ein Modell für die Analyse von Anzeigedaten eingeführt, das an empirischen Befunden (Varianz der Anzeigerate) orientiert ist und die Daten innerhalb der statistisch annehmbaren Fehlergrenzen reproduziert. Die gute Anpassung an die Daten und der kontinuierliche Anstieg in N (jährlich 10-17\%)

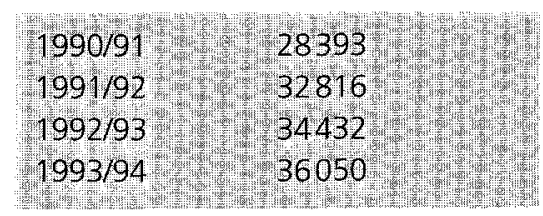

Tabelle 5. Geschätzte Zahl der Konsumenten von Heroin und/oder Kokain für Zweijahresintervalle nach der Peterson-Methode (Quelle: ${ }^{16}$ ). 
unterstützen die Hypothese, dass der Konsum von Heroin und Kokain im Zeitraum 1990-1993 zugenommen hat. Die Bedeutung dieses Befundes im Rahmen der
Evaluation bisheriger Bemühungen zur Eindämmung des Drogenkonsums müsste nun durch Fachleute der Prävention überprüft werden.

\section{Summary}

Increasing number of heroin and cocaine users in Switzerland since 1990

Estimates of the prevalence of deviant behaviour which are based on the usual survey methods, are by far too low. Therefore, the use of capture-recapture methods or of the truncated Poisson distribution is to be preferred, provided appropriate data are available. Here an extended Poisson approach was applied in order to estimate the number of users of hard illegal drugs (heroin, cocaine) in Switzerland for each year from 1990 to 1993 . These estimates indicate an increase by about $50 \%$ during the period $1990-1993$.

\section{Résumé}

La consommation d'heroine et de cocaïne a augmentée en Suisse depuis 1990

Les estimations de la prevalence de la toxicomanie basées sur les méthodes usuelles d'enquête sont largement trop basses. Par conséquent, if faut utiliser des méthodes statistiques plus subtiles. Si des données appropriées existent, on peut exploiter les méthodes capture-recapture ou la distribution de Poisson. Dans cette étude on a estimé le nombre de consommateurs d'heroine et/ou de cocaine en Suisse pour chaque annee de 1990 à 1993, utilisant une distribution de Poisson généralisée. Cette méthode montre une augmentation par $50 \%$ de la consommation des drogues précitées pendant la periode 1990-1993.

\section{Literaturverzeichnis}

1 z.B. Auch wir kommen uns vor wie Sysiphus. Berner Zeitung, 27. 6. 1994. Mehr Repression - gleich viel Konsumenten, Neue Zürcher Zeitung, 15.2.1995. Der Konsum stagniert, die Repression nicht. DrogenMagazin, Februar 1995.

2 Rehm J. Konsumformen und Verbreitung illegaler Drogen in der Schweiz. In: Fahrenkrug, Rehm, Müller, Klingemann, Linder. Illegale Drogen in der Schweiz 1990-1993. Zürich: Seismo Verlag, 1995.

3 Hser Y-I. Population estimates of intravenous drug users and HIV infection in Los Angeles County. Int J Addict 1993; 28:695-709.

4 Simeone RS, Nottingham MS, Holland L. Estimating the size of a heroin-using population: an examination of the use of treatment admissions data. Int J Addict 1993; 28:107-128.

5 Domingo-Salvany $A$, Hartnoll $R$, Maguire A, Suelves J, Anto J. Use of Capture-Recapture to estimate the prevalence of opiate addiction in Barcelona, Spain, 1989. Amer J of Epidemiol 1995; 141 : 567-574.

6 Bundesamt für Statistik (Maag V, Ronez S). Zwanzig Jahre Drogen und Strafrecht in der Schweiz. Statistik aktuell 19, Rechtspflege. Bern 1991.

7 Knolle H. Die Dynamik der Dro genszene in der Schweiz 1977 1990. Soz Präventivmed 1996; 41 : 143-149.

8 Wickens TD. Quantitative Methods for estimating the size of a drug-using population. $\mathrm{J}$ Drug Issues $1993 ; 23: 185-216$.

9 Bundesamt für Statistik (Esterman J, Ronez S). Drogen und Strafrecht in der Schweiz. Zeitreihen zu Ver zeigungen, Strafurteilen und Strafvollzug. Bern 1995.

10 Knolle $H$. Die Entwicklung des Drogenkonsums in der Schweiz seit 1990. Schweiz Zeitschr Volksw u Statistik 1997 (1). im Druck. 
11 Knolle H. Quantitative Methoden der Trendanalyse des Drogenkonsums. Bericht zu Händen des BAG, ISPM der Universität Bern, Bern 1995.

12 Estermann J. Quantitative Analysen. In: Estermann, Herrmann, Hügi, Nydegger. Sozialepidemiologie des Drogenkonsums. Berlin, New York: Verlag für Wissenschaft und Bildung, 1996.

13 Pfanzagl J. Allgemeine Methodenlehre der Statistik II, Berlin, New York: de Gruyter, 1978.

14 Blumenthal S, Dahiya $R$, Gross $A$. Estimating the complete sample size from an incomplete Poisson sample. J Am Stat Assoc 1978; 73 : 182-187.

15 Stuart A, Ord KJ. Kendall's Advanced Theory of Statistics. (Fifth Edition) Vol. 1, London: Griffin \& Co, 1987.

16 Estermann J. Quantitative Analysen. In: Estermann, Herrmann, Hügi, Nydegger. Sozialepidemiologie des Drogenkonsums. Berlin:
Verlag für Wissenschaft und Bildung 1996: 127.

17 Estermann J. Quantitative Analysen. In: Estermann, Herrmann, Hügi, Nydegger. Sozialepidemiologie des Drogenkonsums. Berlin: Verlag für Wissenschaft und Bildung 1996: 153.

18 Estermann J. Quantitative Analysen. In: Estermann, Herrmann, Hügi, Nydegger. Sozialepidemiologie des Drogenkonsums. Berlin: Verlag für Wissenschaft und Bildung 1996: 149.

19 Bundesamt für Gesundheitswesen. Das Drogenproblem im Spiegel der Statistik. In: Bulletin Nr. 29/95. Bern 1995.

20 Knolle $H$. Incidence and prevalence of illegal drug use in Switzerland in the 1980s and early 1990s: an analytical study. Substance Use and Misuse (in press).

21 Stuart A, Ord KJ. Kendall's Advanced Theory of Statistics. (Fifth Edition) Vol. 2, London, 1991.

\section{Danksagung}

Für wertvolle Hinweise zu den sozialmedizinischen Aspekten dieser Arbeit bin ich Herrn Prof. Dr. Theodor Abelin zu Dank verpflichtet. Ebenfalls danke ich Frau Simone Rônez (Bundesamt für Statistik) für die Bereitstellung der Daten.

\section{Korrespondenzadresse}

PD Dr. Helmut Knolle

Institut für Sozial-

und Präventivmedizin

der Universität Bern

Finkenhubelweg 11

$\mathrm{CH}-3012$ Bern

e-mail:knolle@ispm.unibe.ch 


\section{Mathematischer Anhang}

Die Schätzung des Parameters der Poisson-Verteilung ist sehr einfach, wenn das Ereignis, wie in einem klassischen Beispiel von Greenwood und Yule ${ }^{15}$, ein Arbeitsunfall und die Population die Belegschaft einer Fabrik ist. Dann ist $\mathrm{N}$, die Grösse der Population, bekannt, und nur $\alpha$ muss geschätzt werden. Sei $z_{i}$ die Zahl der Personen mit i Ereignissen in einem Jahr ( $\mathrm{i}=0,1$, $2, \ldots)$. Dann führt die Methode der maximum likelihood $\mathrm{zu}$ der folgenden Schätzung, die in einem bestimmten Sinn die bestmögliche Schätzung ist ${ }^{21}$ :

$\alpha=\frac{\sum_{i>0} i z_{i}}{N}$

Wenn es wie hier um Drogenkonsumenten geht, dann ist das Ereignis eine Anzeige, und wir kennen nur $z_{1}, z_{2}, \ldots$, während $z_{0}$ ebenso wie $N$ geschätzt werden muss. Sei m die Zahl der Anzeigen pro angezeigte Person, also

$$
\mathrm{m}=\frac{\sum_{\mathrm{i}>0} \mathrm{i} \mathrm{z}_{\mathrm{i}}}{\sum_{\mathrm{i}>0} \mathrm{z}_{\mathrm{i}}}
$$

Hier steht im Nenner die Zahl der Personen mit mindestens einer Anzeige, und diese ist $\mathrm{N}\left(1-\mathrm{e}^{-\alpha}\right)$. Im Zähler steht die Zahl aller Anzeigen oder $\alpha \mathrm{N}$. Folglich ist $\mathrm{m}=$ $\alpha /\left(1-\mathrm{e}^{-\alpha}\right)$ oder

$\alpha=\mathrm{m}\left(1-\mathrm{e}^{-\alpha}\right)$

Da die $z_{i}(i=1,2, \ldots)$ bekannt sind, kann $m$ sofort berechnet werden, und anschliessend kann man die Gleichung (A3) durch ein iteratives Verfahren mit der Rekursionsgleichung

$\mathrm{x}_{\mathrm{n}+1}=\mathrm{m}\left(1-\mathrm{e}^{-\mathrm{x}} \mathrm{n}\right)(\mathrm{n}=0,1,2, \ldots)$

nach $\alpha$ auflösen. Mit den Daten aus Tabelle 1 erhält man die folgenden Werte von $\mathrm{m}$ und $\alpha$ :

\begin{tabular}{lll} 
& $\mathrm{m}$ & $\alpha$ \\
\hline 1990 & 1,33 & 0,601 \\
1991 & 1,41 & 0,726 \\
1992 & 1,52 & 0,910 \\
1993 & 1,60 & 1,029
\end{tabular}

Mit diesen Werten von $\alpha$ erhält man entsprechende Schätzungen für $\mathrm{N}$, indem man die Gleichung (A1) nach $\mathrm{N}$ auflöst. Die Werte von $\alpha$ liegen jedoch weit über den Populationsmittelwerten der Anzeigerate, die mit dem ZweiGruppen-Modell geschätzt wurden (Tab. 3, letzte Spalte). Infolgedessen liegen die Schätzungen für $\mathrm{N}$ entsprechend tiefer.

Die naheliegende Verallgemeinerung des Poisson-Ansatzes besteht nun darin, dass an die Stelle der einen Rate $\alpha$ eine Verteilung von Raten tritt. Für ein kleines Intervall $\mathrm{dx}$ sei also $\mathrm{F}(\mathrm{x}) \mathrm{dx}$ die Zahl der Personen mit einer Rate zwischen $\mathrm{x}$ und $\mathrm{x}+\mathrm{dx}$. Unter diesen hat die Anzahl

$\frac{x^{i}}{i !} e^{-x} F(x) d x$

genau i Ereignisse zu erwarten. Nun bildet man die Summe über alle $\mathrm{x}>0$ und lässt $\mathrm{dx}$ gegen null gehen. So erhält man für die Zahl der Personen mit i Ereignissen

$\mu_{\mathrm{i}}=\int_{0}^{\infty} \frac{\mathrm{x}^{\mathrm{i}}}{\mathrm{i} !} \mathrm{e}^{-\mathrm{x}} \mathrm{F}(\mathrm{x}) \mathrm{dx} \quad(\mathrm{i}=0,1,2, \ldots)$

Ein geeigneter Kandidat für $\mathrm{F}(\mathrm{x})$ ist z.B. die Gamma-Verteilung

$\Gamma(\mathrm{x} ; \mathrm{r}, \sigma)=\sigma^{\mathrm{r}} \mathrm{x}^{\mathrm{r}-1} \mathrm{e}^{-\sigma} \mathrm{x} /(\mathrm{r}-1) !$

mit den Parametern $\sigma$ und r, wobei $r$ positiv und ganzzahlig ist. Setzt man diese in (A5) ein (die nötigen Formeln der Integralrechnung findet man in jeder mathematischen Formelsammlung) und multipliziert rechts mit $\mathrm{N}$, so folgt:

$$
\begin{array}{r}
\mu_{\mathrm{i}}=\frac{\sigma^{\mathrm{r}}}{(1+\sigma)^{\mathrm{r}+\mathrm{i}+1}} \frac{(\mathrm{r}+\mathrm{i}-1) !}{(\mathrm{r}-1) ! \mathrm{i} !} \mathrm{N} \\
(\mathrm{i}=0,1,2, \ldots)
\end{array}
$$

Die Verteilung (A6) hat den Mittelwert $\mathrm{r} / \sigma$ und die Varianz $\mathrm{r} / \sigma^{2}$. Von allen Gamma-Verteilungen mit gleichem Mittelwert hat diejenige mit $r=1$ die grösste Varianz. Da die empirische Varianz der Anzeigeraten möglicherweise noch grösser ist, wählen wir eine Überlagerung von zwei Gamma-Verteilungen mit verschiedenen Mittelwerten, und zwar:

$$
\begin{aligned}
& \mathrm{D}(\mathrm{x} ; \mathrm{f}, \mathrm{r}, \sigma, \tau)=\mathrm{f} \Gamma(\mathrm{x} ; \mathrm{r}, \sigma) \\
& +(1-\mathrm{f}) \Gamma(\mathrm{x} ; \mathrm{r}, \tau) \quad(0<\mathrm{f}<1)
\end{aligned}
$$

An die Stelle von A7 tritt ein entsprechender Ausdruck. Die Parameter $\mathrm{f}, \sigma, \tau, \mathrm{r}$ sowie $\mathrm{N}$ müssen geschätzt werden. Hier wird jedoch von Anfang an $r=4$ gesetzt, um deren Zahl zu reduzieren. Die Werte von $f, \sigma, \tau$ und $\mathrm{N}$ werden dann so bestimmt, dass die sogenannte Pearson-Statistik

$\mathrm{X}^{2}=\sum_{\mathrm{i}=1}^{\mathrm{k}} \frac{\left(\mathrm{z}_{\mathrm{i}}-\mu_{\mathrm{i}}\right)^{2}}{\mu_{\mathrm{i}}}$

minimal wird. Die entsprechenden Anzeigeraten $\alpha$ und $\beta$ sind die Mittelwerte der Verteilung (A6), wo gegebenenfalls $\sigma$ durch $\tau$ zu ersetzen ist, also:

$\alpha=\sigma / \mathrm{r} \quad \beta=\tau / \mathrm{r}$

Wenn es stimmt, dass die angenommene Verteilung den Daten zugrunde liegt, dann ist $X^{2}$ verteilt wie $\mathrm{chi}^{2}$ mit $\mathrm{k}-4$ Freiheitsgraden, da 4 freie Parameter geschätzt wurden. Da wir die Personen mit 7 und 8 Anzeigen zusammengefasst haben, ist $\mathrm{k}=7$, und folglich hat $\mathrm{chi}^{2}$ 3 Freiheitsgrade. In Tabelle 4 hat $\mathrm{X}^{2}$ für 1990 und 1991 einen Wert, der unter der $95 \%$-Marke $(7,8)$, und für 1992 und 1993 einen Wert, der unter der $99 \%$-Marke $(11,3)$ liegt. Die gute Anpassung an die Daten spricht für die Hypothese, dass die Zahl der Konsumenten zugenommen hat. Ein statistischer Test gegen die Alternativhypothese des konstanten Konsums wird in ${ }^{20}$ gegeben. 